\title{
Evaluation of Erythrocytes Antioxidant Mechanisms in Bovine Babesiosis and Current Advances Treatment in Kaliobea Governorate
}

\author{
Mervat E.I.Radwan ${ }^{1, *}$, Omnea Abdel Hamied ${ }^{2}$, Abdel Fatah Ali $^{3}$ \\ ${ }^{1}$ Department of Veterinary Medicine, Veterinary Hospital, Benha University \\ ${ }^{2}$ Department of Biochemistry, Veterinary medicine, Benha University \\ ${ }^{3}$ Department of clinical Pathology, Veterinary Hospital, Benha University \\ *Corresponding author: Dr_Mervat19@yahoo.com
}

Received May 04, 2013; Revised June 21, 2013; Accepted June 22, 2013

\begin{abstract}
Bovine babesiosis is a febrile, tick-borne disease of cattle and buffalo, caused by one or more protozoan parasites of the genus Babesia.The term "Babesiosis" refers to the acute form. In this study we selected animals from endemic area of Babesia bigemina infection that suffered from fever, emaciated, anemia, icterus and variable weight loss. The acute form is generally characterized by rapid growth and multiplication of the parasite in blood so diagnosis of babesiosis by microscopic examination is applicable in positive infected animals. These ones were distributed in three groups; each group consists of 50 animals and we selected twenty animals healthy (not infected) as control group. The infected animals of the first group was not treated as control positive, the second group was treated with imidocarb antibabesial drug and the third group was treated with imidocarb and Vit $\mathrm{E}$ as antioxidant. A comparison was made between the two methods of treatment by measuring erythrocytes antioxidant mechanisms representing in (G6PD, GR-ase,SOD and MDA) and in plasma (NO and trace element as antioxidant). Blood samples were collected from all animals from jugular vein: two samples, one on anticoagulant to measure erythrocytes antioxidant mechanisms and another to measure NO and trace elements. Results showed increased values of oxidant and decreased values of antioxidant in calves buffaloes suffered from parasitic infection as compared with healthy animals that especially for values of Nitric Oxide (NO) and Malondialdehyde (MDA). So, there was an increase in oxidative markers in animals infected with babisiosis while the levels of antioxidants such as superoxide dismutase(SOD), glutathione reductase (GR-ase); Glucose-6-Phosphate Dehydrogenase(G6PD) decreased, and some trace element showed changes as well (Cu). Addition of antioxidant (Vit E) to treatment decreased oxidative effect of infection and played an important role in increase of antioxidant.
\end{abstract}

Keywords: babesiosis, oxidant, antioxidant and current treatment

\section{Introduction}

Parasites have detrimental effects on host fitness, and infection results in stimulation of the immune system. While defending against infection, the immune system generates toxic oxidants, if oxidants are not sufficiently counteracted by the antioxidant system, a state of oxidative stress will occur. Oxidative stress is an imbalance between radical-generating and radicalscavenging activity, resulting in oxidation products and tissue damage, when the cellular oxidant state is overwhelmed by excess production of reactive oxygen species (ROS),cellular damage occur through oxidative stress and lipid peroxidation (LPO) [1]. Alteration of oxidative stress and malondialdehyde (MDA) is used as biomarkers for free radical -mediated damage [2]. There strong evidence role of erythrocytic peroxidation in pathogenesis of several hemoparastic infections [3]. The nitric oxide (NO) is anther molecule that is free radical mediated in nature, NO mediate host defense mechanism against many intracellular parasitic diseases [4]. In other side GSH is an endogen-derived tripeptide synthesized from the liver and superoxide dismutise (SOD), they are important antioxidant enzymes involved in endogenous antioxidant defense against ROS, these enzymes protect host cells against oxidative damage by reacting with free radicals and peroxides [5]. G6PD has long been recognized as an antioxidant enzyme in erythrocytes, G6PD IS considered the main and only source of NADPH, additionally regulates the activity of enzymes that require NADPH as cofactor, G6PD maintained intracellular glutathione stores when exposed to oxidants because of increased activity of glutathione reductase and increase enzymes activity to confer protection against elevated levels of ROS on cells exposed to oxidant stress [6]. Vitamin E lipid soluble antioxidant can inhibit lipid peroxidation and stabilized membranes with in cells ,vit.E is the only antioxidant that is present in the lipid membrane layer and is also only compound able to break lipid peroxide chain reactions by neutralizing lipid peroxides in addition to oxidants. Vit.E is evidence for decrease MDA level and it is known that vit $\mathrm{E}$ is the first 
line of defense against lipid peroxidation vitamin $\mathrm{E}$ and other hydrophobic antioxidants function mainly in and around the membrane Lipid bilayers, which halt lipid peroxidation through peroxide radical trapping chain breaking antioxidation [7]. So this study is performed to

1-Determination of oxidative stress markers associated with Babesia Bigemina will helpful to clinicians' tracks course of treatment.

2-Antioxidant as vit.E will be helpful for better response in treatment Schedule.

\section{Material and Methods}

This investigation was performed on 20 buffaloes free from babesioes and 150 infected animals examine with clinical signs and case history from endemic area infected with babisia bigemena in farms in Kaliobea governorates and Benha University education veterinary hospital and confirm diagnosis with microscopic examination of blood film for babesia 1/4 - 1/2 inch from the end of the film and transferred from one side of film to other (cross sectional method) to give constant and representative examination according to Barrent (1965) animal can be considered negative if the three slides were negative and determined the degree of parasitaema by recorded the percentage of infected red blood cells in each blood smear as 100 microscopic field were examined.

Infected animals will divided into three groups each group consists of 50 animalss, first group still without treatment as control positive, second group treated with imidocarb(1ml I/M to each $100 \mathrm{~kg}$ body weight and Vitamin E (Tocosel-Forte injection $5 \mathrm{ml}$ dose $1 \mathrm{ml}$ per $50 \mathrm{~kg}$ I/M produced by Pharma Swede-Egypt Vite. E \&selenium) as antioxidant whileThird group treated with imidocarb only. Take 2 samples of blood from all animals first on EDTA to measure antioxidant as(G6PD,GR-ase and SOD;) and oxidative peroxide as MDA as index of lipid peroxidation other samples without anticoagulant to measure serum trace element $(\mathrm{Cu})$ as antioxidant and Nitric
Oxide (NO) as result of lipid peroxidation and compare among four groups, control groups (Healthy and Infected)and treated groups(treated with imidocarb associated with vit.E and imidocarb only)these groups will be compared with healthy group free from infection to knew which group approach from healthy group the second or the third and return to normal state rapidly before other by following the drug pathway after 2 weeks from treatment and notation the results of oxidants and antioxidants parameters which treated group returned to normal state the second group or third group and effect of vit.E as antioxidant in treatment with imidocarb.

\section{Statistical Analysis}

The results were analysed by a one-way analysis of the variance (ANOVA) followed by the Duncan test for multiple comparisons using computer software, Duncan's Multiple Range Test

\section{Results}

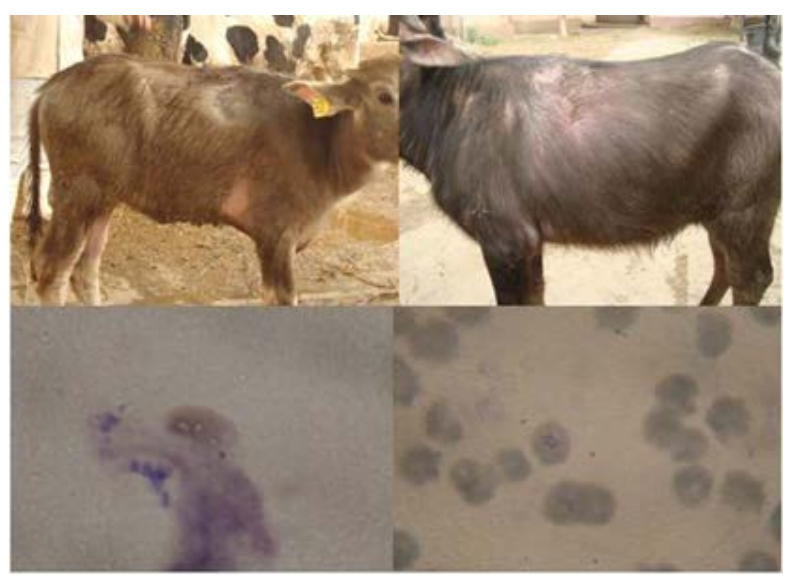

Figure 1. Clinical signs and microscopic examination of calves infected with babesia

Table 1. Effect of infection with babesia Bigemina in buffalo-calves ages 1-3years on concentration of some antioxidants /oxidants markers

\begin{tabular}{|c|c|c|c|c|}
\hline \multirow{2}{*}{$\begin{array}{c}\text { Groups } \\
\text { Antioxidants } \\
\text { And oxidants }\end{array}$} & \multicolumn{2}{|c|}{ Control Groups } & \multicolumn{2}{|c|}{ Treated Groups } \\
\hline & Healthy & Infected & $\begin{array}{l}\text { Imidocarb } \\
\text { and Vit.E }\end{array}$ & Imidocarb \\
\hline GR-ase & $\begin{array}{l}2.577 \pm \\
0.2404^{\mathrm{a}}\end{array}$ & $\begin{array}{l}0.5600 \pm \\
0.1015^{\mathrm{b}}\end{array}$ & $\begin{array}{c}2.130 \pm \\
0.02517^{\mathrm{a}}\end{array}$ & $\begin{array}{c}2.117 \pm \\
0.1260^{\text {ac }}\end{array}$ \\
\hline SOD & $\begin{array}{c}13.13 \pm \\
0.7494^{\mathrm{a}}\end{array}$ & $\begin{array}{l}7.787 \pm \\
.106^{\mathrm{b}}\end{array}$ & $\begin{array}{c}12.00 \pm \\
0.6560^{\mathrm{a}}\end{array}$ & $\begin{array}{l}10.72 \pm \\
0.390^{\mathrm{ab}}\end{array}$ \\
\hline G6PD & $\begin{array}{c}119.5 \pm \\
0.9932^{\text {a }}\end{array}$ & $\begin{array}{l}82.03 \pm \\
8.256^{\mathrm{b}}\end{array}$ & $\begin{array}{l}101.3 \pm \\
3.442^{\mathrm{a}}\end{array}$ & $\begin{array}{l}87.68 \pm \\
5.740 \text { ac }\end{array}$ \\
\hline MDA & $\begin{array}{c}0.8800 \pm \\
0.07767^{\mathrm{b}}\end{array}$ & $\begin{array}{l}2.197 \pm \\
0.2867^{\mathrm{a}}\end{array}$ & $\begin{array}{l}1.111 \pm \\
0.064^{b}\end{array}$ & $\begin{array}{l}1.157 \pm \\
0.029^{\mathrm{ab}}\end{array}$ \\
\hline NO & $\begin{array}{l}62.00 \pm \\
2.517^{c}\end{array}$ & $\begin{array}{l}100.7 \pm \\
1.453^{\mathrm{a}}\end{array}$ & $\begin{array}{l}79.00 \pm \\
2.082^{c}\end{array}$ & $\begin{array}{l}76.00 \pm \\
2.887^{b}\end{array}$ \\
\hline $\mathrm{CU}$ & $\begin{array}{l}99.80 \pm \\
4.933^{\mathrm{a}}\end{array}$ & $\begin{array}{l}80.43 \pm \\
4.523^{b}\end{array}$ & $\begin{array}{l}95.77 \pm \\
4.517^{\mathrm{a}}\end{array}$ & $\begin{array}{l}91.20 \pm \\
1.762^{\mathrm{ab}}\end{array}$ \\
\hline
\end{tabular}

Figure 1 showed emaciated animals with external parasites and blood stain film with geimsa stain showed erythrocytes infected with Babesia and binary fission four cells in RBCs characteristic shape for Babesia differentiated it from other protozoan as Malaria infected RBCs.
Table 1: Values are expressed as mean and mean of standard error ,values with different superscriptions(a,b and $a b)$ in rows differ significantly $(\mathrm{P}<0.05)$.In general infected animals had lower values of antioxidant parameters as(GR-ase,SOD and G6PD) and(Cu) when compared with healthy animals. On other side value of 
oxidant markers (MDA and NO) significant increase in blood of infected group as compared to healthy group. When compare between control groups (negative and infected) and treated groups (treated with Imidocarb associated with vitamin E and Imidocarb only) found significant changes among four groups in antioxidant and oxidant parameters. When compare groups with healthy group found significant different increase oxidants and significant different decrease antioxidants enzymes activity among infected group, treated group with Imidocarb only and non significant different between healthy group and treated group with imidocarb and vitamin $\mathrm{E}$ in antioxidant and oxidant parameters.

\section{Discussion}

Manifestation of oxidative stress occurs due to either inadequate availability of antioxidant enzymes or uncontrolled production of free radicals-induced tissue damage and has been implicated in many pathological conditions. Babesia parasite, likes theleria and malaria parasites, invade erythrocytes of infected animals resulting in destruction of parasitized erythrocytes [8] .The liver plays a central role in babesiosis,It is known as the site where the pre-erythrocytic stages of babesia parasites asexually multiply and where host immature mechanisms develop to fight these pre-erythrocytic stages [9]. In hemoparasitic diseases stimulates the production of various pro-inflammatory cytokines from mononuclear cells in animals and human beings these mediators activate the mononuclear,phagocytic cells to release oxygen and reactive nitrogen intermediates [4,10,11], Current study reveals that infection with babesia bigemina in acute stage(babesiosis) has significant conspicuous increase on erythrocytic LPO and serum NO, in other side it has significant decrease in antioxidant enzymes as(P6PD,GR-ase,DOS) when compare with healthy animals our results were observed that babesia infection has a significant influence on augmented NO production and erythrocytic LPO and excess free radicals leads to oxidative stress. Therefore it could be hypothesized that severity of Babesiosis is related to oxidative stress and loss of body's antioxidant reserve. Augmented erythrocytic LPO and decrease SOD activity have been reported in hemoparasitic infection like Babesia bigemina and Theileria annulated infection respectively [12,13]. who observed that elevated level of plasma NO and enhanced oxidative burst and ROS production by bovine moncytes' and neutrophils during peak of parasitmia [14], $[15,16,17]$. In our study reported that MDA derived from LPO is an important indicator to decide the degree of oxidative damage of cell membrane and it increase when compare with healthy and treated groups. MDA It reacts with cellular membrane elements and results to increase cellular permeability and enzyme activities. The membrane of erythrocytes is rich in polyunsaturated fatty acids and more vulnerable to peroxidative damage.[18,19]. Loads of ROS included Nitric oxide and MDA over burden antioxidant systems induce oxidative stress in the body where major cellular targets of ROS are membrane lipids and Proteins [20]. MDA is high concentration in calves and cows infected with babesiosis [21,22]. Erythrocytes particularly susceptible to an oxidative damage as a result of high polyunsaturated fatty acids content in their membrane and high concentration of oxygen and hemoglobin, hemoglobin is a potentially powerful promoter of oxidative processes [23] .Babesia infection associated with free radical formation by changes antioxidant stress that is very dangerous to erythrocytes [24]. Our results noted that in parasitimic animals showed decrease in antioxidant enzymes as ((P6PD,GR-ase,SOD) and trace element as(Cu) when compare with healthy animals and treated groups . Gr-ase plays a key role in cellular defense against oxidative stress by preventing accumulation of GssG and thus maintaining the redox state [25]. Babesiosis have oxidant antioxidant effect on buffaloes by increase lipid peroxide MDA andNO and decrease antioxidant enzymes and trace element as GSH-PX, SOD, GR-ase and change trace element antioxidant as $\mathrm{Cu}$ and Fe [26]. GSH and GSH-PX represent a major pathway in the cell for metabolizing hydrogen peroxides and lipid peroxides so that the depletion of their level $\backslash$ Activity can lead to accumulation of peroxides increasing them to toxic levels [27]. Babesia showed clean changes in the oxidant, antioxidant markers in blood and increased value of oxidative markers have been implicated in tissue damage and development of chronic diseases [28]. Infected animals with theileriosis showed significant decrease in activities of antioxidant enzymes as SOD, GSH-PX and serum concentration of $\mathrm{Cu}$ were evident in the infected animals in contrast, significantly increase level of MDA [29]. Superoxide dismutase (SOD) is one of the most important antioxidant enzymes present in all oxygen metabolizing cells this enzyme eliminates toxins From body [31]. The antioxidant system consists of antioxidants act by scavenging the radicals directly and sustaining the activity of antioxidant enzymes as inhibiting the activities of oxidizing enzymes in intra erythrocytic parasites theleria annulata and babesia bovis [30]. Animals in our study treated with imidocarb as antbabesial drugs and antioxidant drugs as Vit.E to improve antioxidant mechanisms by increase activity of antioxidant enzymes and scavenger to lipid peroxidation and restoring to normal since Vit.E. reduce and stop metabolism of babesia and strength the membrane of RBCS and decreases haemolysis of it. When compare with healthy animals in our study found there is no significant different between this group and healthy group and found animals after two weeks from administration of treatment appeared healthy, good clinical signs, approach from normal and confirmed this good prognosis by evaluation ((P6PD,GR-ase,SOD) but when compare healthy group and animals which treated antibabesial drug only(imidocarb) found significant different between these group. Antioxidant agent recommendation ,such as vitamin $\mathrm{C}$ and vitamin $\mathrm{E}$ may alleviate oxidative damage, this vitamins are freeradical scavengers and could have a protective effect against oxidative damage and might be better in clinical improvement Vitamine E are free- radical scavengers and has protective effect aganist oxidative stress and oxidative damage and better in clinical improvement [32,31]. Treatment with $\alpha$-Tochopherol increase GSH and glutathione in reduce form has antioxidant properties to inhibits free radical formation and functions more generally as a redox buffer [33]. Pathogenesis of anemia associated with Babesia infection is patialy derived from 
oxidation of the membrane of RBCs so injection of Vit.E may reduce oxidation procedures by increase antioxidants in RBCs [34]. Imidocarb radical cure the infection only But vitamins as E,C and A protect the cells from damage against free radical oxygen generated as a result of parasites and have also protective role in the liver, the effect Vit E as antioxidant appear in high concentrated $\mathrm{O} 2$ as in RBCs may play an important role in keeping stability of erythrocytes (Tocopherols have antioxidant activity as they react with molecular oxygen and free radicals thus prevent autoxidation of polyunsaturated fatty acids in tissues (cellular and sub cellular) [35].

Taking together, the remarkable decrease in the level of antioxidant enzymes activities (G6PD,GR-ase,SOD) and the significant rise in the lipid peroxidation (MDA and NO) ,imply that the babesia bigemina can interfere with the protective antioxidant mechanism of RBCs against oxidative injuries and the antioxidant vitamins were not able to prevent oxidative damage to RBCs membrane .So advise with administration of Vit.E and selenium as antioxidant to compensate shortage and scavenger to oxidative.

\section{Acknowledgment}

To all member stuff of education veterinary hospital Benha University wherever the experiment were performed and stuff of department of infectious diseases special Dr Mohamed hassanin ebeid prof. of infectious diseases Department of Veterinary Medicine.

\section{References}

[1] Saleh.M.A.,O.M, and Al-salahy, M.B., Circulating oxidative stress status in dromedary camels infested with sarcoptic mange. Veterinary Research communication2011. 35, 35-45.

[2] Kuhn,H. and Borchert A. Regulation of enzymatic lipid peroxidation, the interplay of perroxidizing and peroxide reducing enzymes .Free Radical Biology and Medicine 2002. 33,154-172.

[3] Nazifi, S. Razvi,S,M. Mansourian,M . Nikahval,B. and Moghaddam ,M. Studies on correlations among parasitemia and some hemolytic indices in two tropical diseases infers province Iran. Tropical Animals Healthy and Production 2008, 40,47-53.

[4] Brunet, L.R.. Nitric oxide in parasitic infections international immunopharmacology 2001.1, 1457-1467.

[5] Kurt,O,Ok.U.Z.Ertan .P.and Yukset ,H. Antioxidants substances and malaria .Acta Parasitologica Turcica2002. 26,108-112.

[6] Jane ALeopold, Yang-Yi Zhang. Anne W. Scribner, Robert C. Stanton and Joseph Losealzo Glucose-6-Phosphate Dehydrogenase Overexression Decreases Cell Oxidative Stress and Increases Bioavailable Nitric Oxide Arterioscler Thromb Vase Biol (2003): 23:411-417.

[7] Ujjwal Kumar DE, Sahadeb Dey, Partha Sarathi Banerjee, Monalisa Sahoo. Correlations among Anaplasma marginale parasitemia and markers of oxidative stress in crossbred calves Tropical Animal Health and Production (2011)44:3:385-388.

[8] OTSUKa; Y.; Amasaki; M.; Yamato; O.; Maede;Y. The effect of macrophages on the erythrocytes oxidative damage and pathogenesis of anemia in Babesia gibson- infected dogs with low parasitemia J.Vet.Me2002.d . Sci. 64; 221-223.

[9] Cohen,S.Lambert,PH,.InCohen,S.Warren,(EDs) Immunology of parasitic infection ,Second Black Well scientific publication Oxford1982,pp.422-474

[10] Brown,W.C. Shkap,V. Zhu.D., Meguire,T.C. Tuo.W. Meelwain, T.F. and Palmir ,G.H. CD4+ T-Lymphocyte and immunoglobin G2Responses in calves immunized with anaplasma marginale outer membranes and protected against homologues challenge .Infection and Immunity1998 66,5406-5413.
[11] Woldehiwet,Z., The natural history of Anaplasma phagocytophilum, Veterinary parasitology 2010. 167,108-112

[12] Asri Raaei,S and Dalir- Naghadeh B . Evaluation of antioxidant status and oxidative stress in cattle naturally infected with Theileria annulata .Vetrinary parasitology2006. 142, 176-186.

[13] Saleh, M.A, O.M. Mahran and Bassem Al-Salahy,. Circulating oxidative stress status in dromedary camels infested with sarcoptic mange. Vet. Res. Common2010., 35:3 5-45.

[14] Ergonul ,S.and Askar.T.K. The investigation of heat shock protein (HSP27), malondialdhyde (MDA),nitric oxide (NO) and interlukin (IL-6.IL-10) levels in cattle with anaplasmosis Kafkas University Vetrinary Fakultesi Dergisi2009 .15,575-579.

[15] Park,J.and Rikihisa,Y. L.Arginine -dependant Killing of intracellular Ehrlichiaristicii by macrophages treated with gamma interferon .Infection and Immunity1992, 60,3504-3508.

[16] Waytt,C.R., Davis,W.C., Knowles,D.P., Goff, W.L., Palmer,G.H. and McGuire, T.C., Effect on intraerythrocytic Anaplasma marginale of soluble factors from infected calf blood mononuclear cells, Infection and immunity1996., 64.4846-4849.

[17] Court,R.A.Jackson L.A. and Lee,R.P. Elevated anti-parasite activity in prepheral blood monocytes and neutrophils of cattle infected with babesia bovis .International Journal of Parasitology2001 19,29-37.

[18] Sato.y..Kanazawa,S.Sato.K.and Suzuki,Y.,.Mechanism of freeradical induced hemolysis of human erythrocytes:II.Hemolysis by lipid soluble radical initiator, Biological and Pharmaceutical Bulletin1998 ,21.250-256.

[19] Bolchoz L.I.Morrow. J.D.Jollow.D.J.and McMillan,D.C., Primaquine -iduced hemolytic anemia ,effectof 6-methoxy -8hydroxylaminoquinoline on rat erythrocyte sulfhydrylstatus .membrane lipids ,cytoskeletal, proteins and morphology. The journal Pharmacology and Expermintal Therapeutics2002, 303,141-148.

[20] Tsukahara, H.,. Biomarkers for oxidative stress: clinical application in pediatric medicine. Curr. Med. Chem. 2007, 14:33951.

[21] Sedar, D.D. Yeter, B. Kamile and N.A.G. Ozdaland,. Status of Lipid Peroxidation, Antioxidants and Oxidation products of Nitric oxide in Equine Babesiosis: Status of Antioxidant and Oxidant in Equine Babesiosis. J. Equi. Vet. Sci. 2009, 29:743-774.

[22] Stich, R.W., L.K.M. Shoda, M. Dreewes, B. Adler, T.W. Jungi and W.C. Brown,. Stimulation of nitric oxide production in macrophages by Babesia bovis. Infection and Immunity 1988, 66:4130-4136.

[23] Cimer; M.Y.: Free. radical metabolism in human erythrocytes. Chin. Chim. Acta2008; 390: 1-11

[24] Celi, P., Biomarkers of oxidative stress in ruminant medicine., Immunopharmacol Immunotoxicol. 2010, Sep 18.

[25] Muller,J.G, Bucheler,U.S. Kayser, K.,schrimer,R.H.,Werner,D. and Krauth.siegel, RGlutathione reductase in human and murine lung tumors, high levels of m RNA \& enzymatic activity.cell. Mol.Biol.1993 .39, 389-396.

[26] EL.Moghazy, F.M Effect of parasitic infection on oxidant \Antioxidant status in Buffaloes Middle-East Jornal Scientific. Research2011 7(4): 585-593.

[27] Genc ,S.,Gurdol,F.Oner-Iyidogan,Y.and Onaran,I ., The effect of melatonin administration on ethanol iduced lipid peroxidation in rats Pharmacological Research 1998 ,37:37-40.

[28] Olmedilla, B., F. Granado, S. Southon, Al. Wright, I. Blanco, E. Gil-Martinez, H. van den Berg, D. Thurnham, B.Corridan, M.Chopra and I. Hininger, A European multicentre, placebocontrolled supplementation study with alpha-tocopherol, carotenerich palm oil, lutein or lycopene: analysis of serum responses. Clin. Sci. Lond 2002. , 102:447-56.

[29] Nazifi,S, Razvi S.M. Kianiamin P, Rakhshandehroo E .Evaluation of erythrocyte antioxidant mechanisms: antioxidant enzyme Lipid perixidations and serum trace element associated with progressive anemia in ovine maligant theileriosis. Parasitol Res.2011 Aug: $\log (2):$ 275-281.

[30] Azeemi, S.T., S.M. Raza, M. Yasinzai and A.Samad, Effect of differant wavelenths on superoxide dismutase. J. Acupunct Meridian Stud 2009, 2:236-8.

[31] Ali Hassanpour, Yousef Chader Sabegh and Ali Sadeghinasab.Assessment of serum aaaaantioxidant enzymes activity in cattle suffering from Theileriosis.European Journal of Expermintal Biology 2013, 3(1) : 493-496. 
[32] Kamil BicEK, Yeter DEGER \& Serdar DEGER Some biochemical and hematological parameters of sheep infected with babesia species YYU Vet Derg2005, 16(1) 33-35.

[33] Nadia, Z. Shaban, Madiha, H. Helmy, Mohmed, A.R.E Kersh; Bothaina, F. Mohmed. Effect of Bacillus Thuringiensis toxin on hepatic lipid peroxidation and free radical scavengers in rats given alpha-tocopherol or a catylsalicy, comparative Biochemistry and Physiology part,(2003 C135:405-414.

[34] Yukio ya gI, Prasobporn ThoNgNo oN, Hiroki Shiono and Yukio Chik AyAMA, Increase in oxidized proteins in Theileria-infected erythrocytes membrane.J.Vet.Med.Sci2002 .64(7):623-625.

[35] Henri J.Vital\&A. Gorenflot. chmotherapy against veterinary parasitology 2006 138,147:160. 